\title{
Can gold nanoparticles improve delivery performance of polymeric drug-delivery systems?
}

\author{
Elisa Lacroce ${ }^{1}$, Paola Saccomandi ${ }^{\text {(D) } \& \text { Filippo Rossi*,1 }}$ \\ ${ }^{1}$ Department of Chemistry, Materials \& Chemical Engineering 'Giulio Natta', Politecnico di Milano, via Mancinelli \\ 7, Milan 20131, Italy \\ ${ }^{2}$ Department of Mechanical Engineering, Politecnico di Milano, via La Masa 1, Milan 20156, Italy \\ *Author for correspondence: Tel.: +39 022399 3145; filippo.rossi@polimi.it
}

"In this Editorial the applications of Au NPs and thermo-responsive hydrogels are summarized in order to underline the importance of these hybrid materials in the biomedical field."

First draft submitted: 10 May 2021; Accepted for publication: 20 May 2021; Published online:

27 May 2021

Keywords: colloids • drug delivery • gold nanoparticles • thermoresponsive

During the recent developments in nanomedicine, gold nanoparticles (Au NPs) have emerged as interesting tools in the field of drug-delivery systems (DDS) due to their low toxicity, stability, easy synthesis and reproducibility [1]. $\mathrm{Au}$ NPs are able to link therapeutic molecules on their surface by covalent or noncovalent bonding or by a previous functionalization of Au NPs and release the drug only in a specific site without damaging the healthy tissue [2]. Moreover, the small size of gold NPs allows their accumulation in sites of tumor and inflammation and exhibit fast cell uptake using mechanisms different from those typical of small molecules [3]. Their optical property is characterized by surface plasmon resonance (SPR), by which incident light is converted into heat, which makes them very useful in promoting photothermal drug release [4]. The main drawback resides in the fact that after their administration, if not confined, they escape through the circulatory torrent without reaching the target site and so losing their efficacy. For this purpose, hybrid materials formed by the loading of Au NPs inside polymer networks are studied to improve local drug delivery. In this case, the easy injection and confinement of the hydrogel in the site of injury represent the main advantages together with local release of both small and large molecules in response to thermal stimuli [5].

\section{Properties of Au NPs}

Au NPs are investigated in the biomedical field because of their biocompatibility, easy synthesis and easy functionalization, together with their magnetic and optical properties [6]. Their nano size allows a high surface area to volume ratio leading to the possibility of a high surface functionalization and interactions with the biological environment. Their functionalization takes place in two steps: first using thiolate molecules on the surface and then through second bonding between free molecules and the pregrafted thiolates [7]. This second bond can be of covalent type, such as by using maleimide, carbodiimide and click chemistry [3] or noncovalent, such as electrostatic or hydrophobic interactions. In particular, the use of covalent conjugation is justified by the using of Au NPs as targeting or imaging contrast agents, while noncovalent conjugation can be used for drug-delivery purposes because stimuli coming from the external environment can promote the release of the attached molecules.

A typical property of Au NPs is the ability of changing the color of their aqueous solution depending on their size (from 1 to $100 \mathrm{~nm}$ ), but the main and useful one is to convert an electromagnetic field of light into heat. This phenomenon is caused by the optical property of SPR, by which the excited surface electrons of Au NPs thermalize with the phonons of the NP releasing heat $[8,9]$. Au NPs are characterized by the tunability of the SPR, which depends on the Au NPs shape, size and aspect ratio. Interactions between phonons then transfer the heat from NPs to the external environment [10]. When the shape of Au NP changes from sphere to rod, a change of 
the absorption and scattering cross-section occurs, with a shift of the absorption co-efficient from the visible to the near infrared (NIR) spectral range. This characteristic of the Au NPs allows wavelength-selective light absorption and consequent temperature increase in the NIR region, where the optimum wavelength for light penetration in biological media is achieved.

\section{Thermo-responsive polymeric systems}

Polymeric particles are colloidal DDS able to encapsulate or link drug molecules with the principal aim to protect them from renal clearance, immune response and degradation in order to improve biodistribution inside the body. The widely known mechanisms of passive and/or active targeting allow the accumulation of polymeric NPs inside the tissue to be treated, where specific environment conditions lead to the release of the drug from NPs. For example, tumor tissues are characterized by a lower $\mathrm{pH}$ and a temperature $1-2^{\circ} \mathrm{C}$ higher than that of healthy tissue [11]. In other cases, an external heat source can be applied in a specific site of the body to create a local increment of temperature. Thermo-responsive colloids are suitable for this purpose.

In particular, one of the most studied micelles in the drug delivery field is represented by $\mathrm{N}$-isopropylacrylamide (NIPAM) monomer, which is characterized by a lower critical solution temperature (LCST) of $32^{\circ} \mathrm{C}$, above which it becomes less soluble. In addition, the copolymerization of NIPAM with hydrophilic monomer (i.e., lactic acid) results in an increase of the resultant polymer's LCST, whereas the addition of hydrophobic monomer leads to a lower LCST. In the majority of cases, at temperatures below the LCST, the micelle presents the NIPAM blocks in its shell and other hydrophobic blocks in its core, but when the temperature overcomes the LCST the NIPAM becomes less soluble and collapses leading to a change of micellar structure with consequent release of the encapsulated molecule $[12,13]$. Polymeric NPs containing NIPAM in the copolymer use the same principle to release the encapsulated drug in combination with a $\mathrm{pH}$-responsive delivery [14].

Another strategy is represented by thermoresponsive block copolymers, such as that formed by ethylene glycol methyl ether methacrylate and propylene glycol methacrylate, that can be used to enhance drug delivery performances and cell uptake for temperatures higher than $37^{\circ} \mathrm{C}$ [15]. In this case the coating onto NPs can swell or shrink depending on temperature and this behavior influences cell uptake and so selective drug-delivery properties. Finally, thermo-responsive hydrogels represent another case of polymeric DDSs that contract at temperature higher than LCST [16]. The shrinking of hydrogel network causes the leakage of the water where the therapeutic agent is solved. One of the main advantages is the possibility to minimize the risks of surgical procedures due to their injectability and ability to create a $3 \mathrm{D}$ network in situ, in the target tissue.

\section{The new frontier: hybrid materials}

In the last decades, hybrid materials based on the union of Au NPs and polymers attracted a lot of interest in biomedical field, although their studies are still limited. In particular, they combined the optical property of Au NPs with the thermo-responsive property of hydrogels. For example, the research group of Ying Qu et al. [17] developed a hybrid material to prevent the postoperative recurrence of breast cancer. Gold nanorods were embedded inside a hydrogel containing NIPAM in order to promote a photothermal release of doxorubicin after the excitation with a near-infrared laser. The effect of the change in temperature inside the hydrogel led to a contraction of the thermo-responsive hydrogel and the consequent release of drug.

In another study, Au NPs embedded cellulose grafted polyacrylamide hydrogels were used to improve thermal and rheological properties of the hydrogels together with a sustained and controlled long term release of ciprofloxacin drug [18]. The improved mechanical properties may be present due to strong interactions between Au NPs and polymer chains that can provide high crosslinking strength.

Finally, a recent work of the research group of Rossi [5] is based on a hybrid material composed by Au NPs loaded in a hydrogel of agarose and carbomer 974P with the final aim to control the release of therapeutic molecules by using an external UV light. In comparison with the thermo-responsive hydrogels described above, this hydrogel responds in the opposite way: the heat released from Au NPs promotes the relaxation and widening of the hydrogel's mesh. Consequently, physically entrapped molecules can diffuse outside the polymer network. Another interesting point underlined is the key role of Au NPs aggregation that can influence network relaxation after light-based stimuli and consequently release rate. In particular they observed a faster release of a mimetic drug from the hydrogel loaded with uncoated Au NPs than that loaded with PEGylated Au NPs due to the aggregation of the NPs inside the hydrogel. Therefore, by combining Au NPs with polymeric hydrogels it is possible to have an injectable 
DDS that guarantees a local and controlled drug delivery by applying an external excitation source, limiting in this way the adverse effects derived from the accumulation of drugs in different organs of the body.

\section{Summary}

Au NPs are of particular interest in the biomedical field, especially for their size and shape dependent properties. In particular, their optical properties can be used to enhance the local and controlled delivery of drug from a thermo-responsive hydrogel. In this Editorial the applications of Au NPs and thermo-responsive hydrogels are summarized in order to underline the importance of these hybrid materials in the biomedical field.

\section{What about the future?}

The main challenge in the use of Au NPs in thermal therapy is represented by the delivery of an optimized concentration of NPs in the target site, in order to be high enough to take advantage of the thermal effect but considering also the necessity to reduce laser intensity directed to the healthy tissue.

In this framework, attention should be given to engineering that can help to plan and predict the settings of $\mathrm{Au}$ NPs-based thermal therapy, together with intraprocedural monitoring of the thermal outcome. This could support the design and the proper control of the therapy. Indeed, a good understanding of the phenomena involved in laser-tissue interaction with Au NPs is fundamental for the design of the preplanning treatment and to define an accurate thermometric approach.

\section{Financial \& competing interests disclosure}

The authors have no relevant affiliations or financial involvement with any organization or entity with a financial interest in or financial conflict with the subject matter or materials discussed in the manuscript. This includes employment, consultancies, honoraria, stock ownership or options, expert testimony, grants or patents received or pending, or royalties.

No writing assistance was utilized in the production of this manuscript.

\section{References}

1. Amina SJ, Guo B. A review on the synthesis and functionalization of gold nanoparticles as a drug delivery vehicle. Int. J. Nanomed. 15, 9823-9857 (2020).

2. Kumar A, Zhang X, Liang XJ. Gold nanoparticles: emerging paradigm for targeted drug delivery system. Biotechnol. Adv. 31(5), 593-606 (2013).

3. Dreaden EC, Alkilany AM, Huang X et al. Nanomedicine themed issue the golden age: gold nanoparticles for biomedicine. Chem. Soc. Rev. 41, 2740-2779 (2012).

4. Wang Y, Kohane DS. External triggering and triggered targeting strategies for drug delivery. Nature Rev. Mater. 2(6), 1-14 (2017).

5. Moretti L, Mazzanti A, Rossetti A et al. Plasmonic control of drug release efficiency in agarose gel loaded with gold nanoparticle assemblies. Nanophotonics 10(1), 247-257 (2021).

6. Wahid F, Zhao XJ, Jia SR et al. Nanocomposite hydrogels as multifunctional systems for biomedical applications: current state and perspectives. Compos. B Eng. 200, 1359-8368 (2020).

7. Yeh YC, Creran B, Rotello VM. Gold nanoparticles: preparation, properties, and applications in bionanotechnology. Nanoscale 4(6), 1871-1880 (2012).

8. Ahmadi TS, Logunov SL, El-Sayed MA. J. Phys. Chem. 100(20), 8053-8056 (1996).

9. Asadi S, Bianchi L, De Landro M et al. Laser-induced optothermal response of gold nanoparticles: from a physical viewpoint to cancer treatment application. J. Biophotonics 14(2), e2020001 (2021).

10. Jain PK, Huang X, El-Sayed IH et al. Noble metals on the nanoscale: optical and photothermal properties and some applications in imaging, sensing, biology, and medicine. Acc. Chem. Res. 41(12), 1578-1586 (2008).

11. Vaupel P, Kallinowski F, Okunieff $\mathrm{P}$ et al. Blood flow, oxygen and nutrient supply, and metabolic microenvironment of human tumors: a review. Cancer Res. 49(23), 6449-6465 (1989).

12. Qu T, Wang A, Yuan J et al. Preparation and characterization of thermo-responsive amphiphilic triblock copolymer and its self-assembled micelle for controlled drug release. Colloid Surf. B 72(1), 94-100 (2009).

13. Liu SQ, Tong YW, Yang YY. Incorporation and in vitro release of doxorubicin in thermally sensitive micelles made from poly(N-isopropylacrylamide-co-N,N-dimethylacrylamide)-b- poly(D,L-lactide-co-glycolide) with varying compositions. Biomaterials 26(24), 5064-5074 (2005).

14. Lo CL, Lin KM, Hsiue GH. Preparation and characterization of intelligent core-shell nanoparticles based on poly(D,l-lactide)-g-poly(N-isopropyl acrylamide-co-methacrylic acid). J. Control. Rel. 104(3), 477-488 
15. Abulateefeh SR, Spain SG, Thurecht KJ et al. Enhanced uptake of nanoparticle drug carriers via a thermoresponsive shell enhances cytotoxicity in a cancer cell line. Biomater. Sci. 4(1), 434-442 (2013).

16. Klouda L, Mikos AG. Thermoresponsive hydrogels in biomedical applications. Eur. J. Pharm. Biopharm. 68(1), 34-45 (2007).

17. Qu Y, Chu BY, Peng JR et al. A biodegradable thermo-responsive hybrid hydrogel: therapeutic applications in preventing the post-operative recurrence of breast cancer. NPG Asia Mater. 7, e207 (2015).

18. Prusty K, Swain SK. Release of ciprofloxacin drugs by nano gold embedded cellulose grafted polyacrylamide hybrid nanocomposite hydrogels. Int. J. Biol. Macromol. 126, 765-775 (2019). 